\title{
Location and Capacity Manufacturing Calculation of Electric Vehicle Charging Station Based on Queuing Theory
}

\author{
QianQian Mao ${ }^{1}$, Jianli Yang ${ }^{1}$, Zheqiong Pan ${ }^{1}$, Kelong Liu ${ }^{2}$, Yang Jiao ${ }^{2}$ \\ ${ }^{I}$ Ningbo power supply company of State Grid Zhejiang Electric Power Co., Ltd, Ningbo 315100, China \\ ${ }^{2}$ State Grid Zhejiang Ningbo Zhenhai Power Supply Co., Ltd, Ningbo 315100, China \\ Corresponding author.
}

\begin{abstract}
With the rapid development of industry and manufacturing, the world vigorously promotes green environmental protection, the construction of charging station is particularly important. It is urgent to study the location and capacity of charging station. In this paper, we will analyze the charging behavior of electric vehicles, and combined with the queuing theory model of electric vehicles, optimize the number of chargers by using the minimum objective function of the comprehensive cost of building charging stations. According to the maximum access power allowed by the distribution network, the service radius and scale of the target charging station are finally determined, and then 0-1 planning is used to finally determine the construction site. At the end, we give a specific example analysis to verify the effectiveness of the research results.
\end{abstract}

Keywords: Queuing Theory, Location, Capacity Manufacturing, 0-1 planning

\section{Introduction}

The automobile has a history of one hundred years from its invention to the present. It has brought many benefits. It has brought the convenience of transportation and travel for human beings. It also brings comfort to humans. But while the car has brought huge benefits to mankind, it also brings many disadvantages and challenges. Environmental pollution is the most serious drawback. Car exhaust emissions make the earth warm and pollute the air and endanger people's health; sound pollution is also very serious. The sound of car engines and horns makes local users uneasy and emotional; energy The consumption problem is also becoming more and more serious. The global oil resources are very limited. After hundreds of years of consumption, mankind must consider and study the future resources.

Electric vehicles with huge advantages in environmental protection have gradually entered the field of vision of mankind and have become research targets and development targets. Electric vehicles use environmentally friendly and pollution-free green energy, which can reduce energy consumption and environmental pollution for the world. Therefore, the promotion and development of electric vehicles is very important and necessary.

The development of automobiles will be greatly accelerated. A lot of research has been done on the planning and construction of charging stations at home and abroad. Tang proposed the method of variance analysis in the article [1], and concluded that mileage, electric vehicle distribution and passenger distribution are factors that affect the location of electric vehicle charging stations. Narges-Shahraki [2] proposed an optimization model based on the driving mode of the vehicle. On the basis of meeting the public charging demand, it selects a reasonable location of the public charging station to maximize the mileage. Wang Meng [3] proposed a multi-level fuzzy method to evaluate the location of charging stations; Fang He [4] constructed an extended network structure, using this structure to establish an effective charging strategy model for electric vehicle drivers, and combined The approximate queuing time function establishes a variational inequality to describe the equilibrium path selection and charging behavior of electric vehicles. Ren Xianqiang [5] took the genetic algorithm as the goal, established a site selection model with the smallest total social cost, and constructed an evaluation index system based on 5 ISSN: 0010-8189 
factors affecting site selection. Jia Xu [6] established an electric vehicle charging station project evaluation system through 15 sub-standards, and constructed a charging station planning model using BP neural network to predict the location and capacity of the charging station planning. Wang Jingmin [7] proposed a new hybrid model, which is used to determine the location of charging stations, using a mobile refueling location model and a queuing theory model, and a robust optimization algorithm to solve the location model. Chen Rui [8] proposed a two-layer mathematical model to derive the optimal design. This method solves the balance problem between path selection and charging waiting time. Xue Bai [9] proposed a hybrid evolutionary algorithm combining non-dominant sorting genetic algorithm II with linear programming and neighborhood search.

In this paper, we will analyze the charging behavior of electric vehicles, establish the capacity of charging stations and determine the construction site. At the end, we give a specific example analysis to verify the effectiveness of the research result.

\section{Research on Location and Capacity of Electric Vehicle Charging Station}

\subsection{Charging station location}

The central place theory $[10,11]$ was proposed by Kristaller. The center is a place that provides services to surrounding customers, which can be as small as a convenience store, or as large as a city. The central place theory takes the central place as the center, analyzes the formation process of the market area, and derives the hexagonal market area structure, which is a classic theory of the service industry layout.

\subsubsection{Service Scope and Threshold}

The service area of the center can be described as a circle. The radius of the circle is the service area of the service center, because this radius is the farthest distance that the customer is willing to come and receive the service. If the distance is exceeded, the user will go to other service areas. The service center needs to have income to maintain the entire service cost, so a certain amount of customers need to come to receive services and pay fees. The residential area of these customers is the threshold distance.

\subsubsection{Hierarchy}

The types of services provided by the service center have different levels. If the service center's service is better and higher-end, then this service center is an advanced service center, and its service range will be very broad, and there will be very few such service locations in the entire region. On the contrary, it is a low-level service center, which has a large number of areas and a very small service area.

\subsubsection{Hexagonal structure}

Although the central service area of the theory was originally a circular area centered on the service center. with the emergence of multiple service facilities and competition, the gap between the circles was occupied and gradually became a Hexagonal structure of honeycomb shape.

Comparing a charging station to a service center, its service radius should meet the convenience of electric vehicle users, so we use the maximum time for electric vehicle users to find a charging station to determine the service radius. In addition to the user's time and speed, we should also consider the degree of congestion on the road, the degree of traffic congestion will affect the user's actual driving distance per unit time.

Assume that the maximum time takes a user to go to an electric vehicle charging station is $T$; The average speed of electric vehicle users is $V$; The traffic impedance coefficient is $\alpha$. Then the calculation formula of the service radius of the charging station is: 


$$
R=\frac{T V}{\alpha}
$$

\subsection{Queuing theory}

In our lives, we have all encountered a service center with a large number of people, which exceeds the capacity of the service center, so we will wait in line for service. In order to solve some problems of queuing and improve efficiency, a queuing service system theory has been formed, which is called queuing theory. Queuing theory conducts statistical analysis and data processing on customer arrival time intervals and service time, and obtains system indicators. These indicators include queuing length, stay time, waiting time, service intensity, and so on. The model of the queuing process is shown in Figure 1. Customers queue up from the customer source to the service organization according to the queuing rules, and leave after the service is completed.

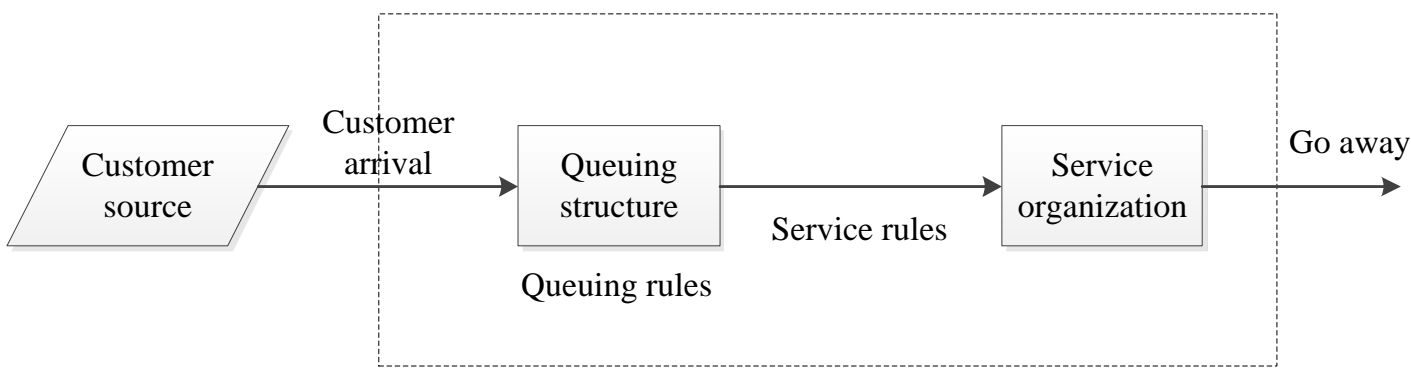

Figure 1 Queuing process model

When you encounter an actual queuing problem, you need to study its queuing model. The time interval distribution of customers arriving at the service organization and the time distribution of customers being served need to be determined by statistical analysis of actual data. Therefore, the basic quantitative indicators need to be determined first:

(1)Captain: The number of customers in the system, the expected value is recorded as $L_{s}$.

(2)Queue length: the number of customers waiting in line in the system, the expected value is recorded as $L_{q}$.

(3)Stay time: the time a customer stays in the system, the expected value is recorded as $W_{s}$.

(4)Waiting time: the time a customer waits in the system, the expected value is recorded as $W_{q}$.

The basis for calculating these indicators is the probability of expressing the state of the system. The status of the system refers to the number of customers in the system, and its value may be:

(1) When the number of teams in the system is unlimited, $n=0,1,2 \ldots \ldots$.

(2)When the number of teams in the system is limited (the maximum is $N$ ), $n=0,1,2, \ldots ., N$.

(3)When the customer is unwilling to wait, then if the number of service desks is $c, n=0,1,2, \ldots \ldots, c$.

Because the probability of the state changes over time, So we use $P_{n}(t)$ to indicates the probability that the system state is $n$ at time $t$. The method of obtaining the state probability is to establish the differential difference equation of $P_{n}(t)$ and t.

The standard $M / M / c$ model satisfies the following conditions:

(1)The source of customers is unlimited, the arrivals of customers are independent of each other, the number of arrivals within a time period obeys the Poisson distribution, and the process is stable.

(2)Single team, first-come, first-served, unlimited team leader.

ISSN: 0010-8189

(C) CONVERTER 2020

www.converter-magazine.info 
(3)With multiple service desks, the service time of each customer obeys a negative exponential distribution (i.i.d). (4)Inter-arrival time and service time of customers are independent of each other.

(5)Each service desk is independent of each other and the average service rate is the same $\mu_{1}=\mu_{2}=\cdots=\mu_{c}=\mu$ 。 Therefore, the average service rate of the service organization is $n \mu(n<c)$ or $c \mu(n \geq c)$.

The transition relationship between the states of the queuing system is shown in Figure 2. State 1 transitions to state 0 , indicating that the transfer rate of a customer in the system being serviced is $\mu P_{1}$. State 2 to state 1 means that one of the clients on the two service desks has been serviced and left, and the state transfer rate is $2 \mu P_{2}$. By analogy, consider the transition from state $\mathrm{n}$ to $\mathrm{n}-1$. When $n \leq c$, the state transfer rate is $n \mu P_{n}$; when $n>c$, there are only c service desks and at most c clients are served, so the state transfer rate can only be $c \mu P_{n}$. Suppose $\rho=\frac{\lambda}{c \mu}$, which is called the average utilization rate of the system service organization. Only when $\rho<1$ does not form an unlimited queue.
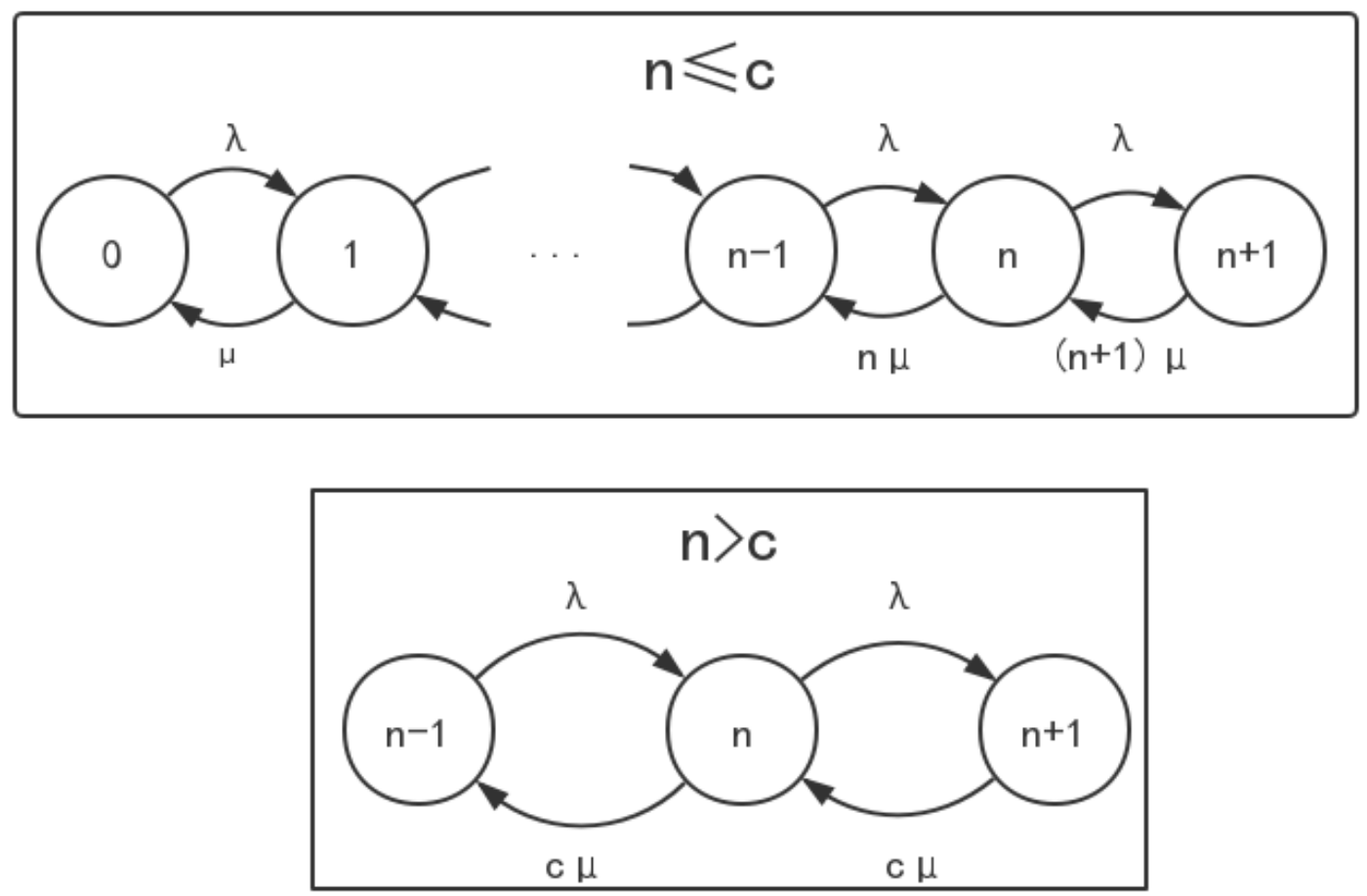

Figure 2 Schematic diagram of state transition relationship

According to Figure 2, we can get:

$$
\left\{\begin{array}{l}
\mu P_{1}=\lambda P_{0} \\
(n+1) \mu P_{n+1}+\lambda P_{n-1}=(\lambda+n \mu) P_{n}(1 \leq n \leq c) \\
c \mu P_{n+1}+\lambda P_{n-1}=(\lambda+c \mu) P_{n}(n>c)
\end{array}\right.
$$


Here $\sum_{i=0}^{\infty} P_{i}=1, \rho \leq 1$. Solving formula (2) by recursive method, the state probability can be obtained:

$$
\left\{\begin{array}{l}
P_{0}=\left[\sum_{k=0}^{c-1} \frac{1}{k !}\left(\frac{\lambda}{\mu}\right)^{k}+\frac{1}{c !} \bullet \frac{1}{1-\rho} \bullet\left(\frac{\lambda}{\mu}\right)^{c}\right] \\
P_{n}=\left\{\begin{array}{l}
\frac{1}{n !}\left(\frac{\lambda}{\mu}\right)^{n} P_{0}(n \leq c) \\
\frac{1}{c ! c^{n-c}}\left(\frac{\lambda}{\mu}\right)^{n} P_{0}(n>c)
\end{array}\right.
\end{array}\right.
$$

Finally, the following system indicators are obtained:

$$
\left\{\begin{array}{l}
L_{q}=\sum_{n=c+1}^{\infty}(n-c) P_{n}=\frac{(c \rho)^{c} \rho}{c !(1-\rho)^{2}} P_{0} \\
L_{s}=L_{q}+\frac{\lambda}{\mu} \\
T_{q}=\frac{L_{q}}{\lambda} \\
T_{s}=\frac{L_{s}}{\lambda}
\end{array}\right.
$$

Where:

$L_{q}$ - The average queue length of the system;

$L_{s} \longrightarrow$ System average captain;

$T_{q}$ - The average waiting time of customers in the system;

$T_{s} \_$The average time the customer stays in the system.

\subsection{Application of queuing theory in the capacity of charging station}

Due to the different economic developments in various regions, the distribution of electric vehicles in various regions is not uniform. For research needs, electric vehicles can be distributed approximately evenly in the area. Set its areal density as $\mathrm{f}$, then it is in a circle with $R$ as the radius. The number of electric vehicles in the area $N$ is:

$$
N=\pi R^{2} \mathrm{f}
$$

Assuming that the average annual mileage of electric vehicles is L, the energy consumption per 100 kilometers is $W_{100}$, the average annual effective driving days is $d$, and the battery capacity is $G$, then the number of vehicles arriving per unit time $\lambda$ is: 


$$
\lambda=N \frac{L W_{100}}{100 d 24 G}
$$

Since electric vehicles accept charging services at random, whether different users accept services is independent of each other. It obeys the "first come, first served" rule. The time interval for users to reach the charging station obeys the Poisson distribution with a parameter of $\lambda$. Electric vehicles accept The service time of the charger obeys the negative exponential distribution of parameter $\mu$, where $\mu$ is the service rate of the charging pile. At this time, the queuing system model is the standard $M / M / c$ model, and the balance equation of the electric vehicle charging station service system can be obtained as (2).

Where:

$P_{n}$ - Indicates the probability that $\mathrm{n}$ electric vehicles in the service system will receive charging services, and $\sum_{i=0}^{\infty} P_{i}=1$

$\mathrm{n}$ - Is the number of electric vehicles in the charging service system;

$\mathrm{c}-\mathrm{I} s$ the number of charging piles.

Let $\rho=\frac{\lambda}{c \mu}$ be called the utilization rate of the service organization, and it can also be called the utilization rate of the charging facility. Solve the difference equation with the recursive method, the state probability can be obtained as equation (3), then the operating index of the system can be obtained as equation (4).

Where:

$L_{q}$ - The average queue length of the charging service system;

$L_{s} \_$Average captain of charging service system;

$T_{q}$ The waiting time of electric vehicles in the system;

$T_{s} \_$The length of time the electric vehicle stays in the system.

\subsection{Optimization of queuing system}

Generally speaking, improving the quality of service will reduce the waiting cost of customers, but it will also increase the cost of the service center, so we need to consider both changes to optimize the system, as shown in Figure 3. There are two commonly used optimization objective functions:

(1)Minimize the sum of service agency costs and customer waiting costs.

(2)Maximize net income or profit, that is, maximize the difference between service revenue and service cost. 


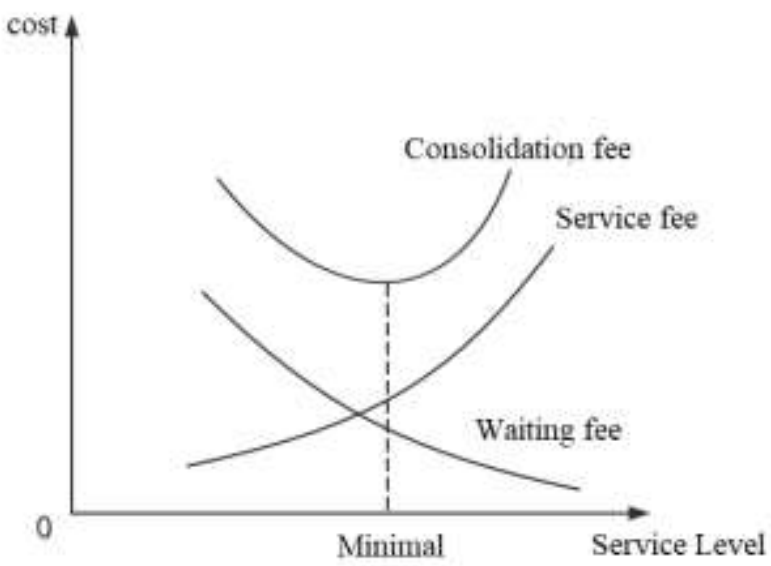

Figure 3 Relationship between cost and service level

Costs are considered in terms of unit time under steady-state conditions. Generally speaking, service costs can be estimated, but customer waiting costs are different. If the assembly line machine fails, the production loss can be estimated. However, it is difficult to estimate the loss of some patients who have aggravated their condition due to delays in waiting for treatment, but it can be estimated by statistical experience data.

The following represents the service level:

(1)Average service rate $\mu$.

(2)Service equipment, such as the number of service desks $c$, the maximum number of queues $N$, and the service level is represented by the service intensity $\rho$.

The above solution methods are mainly based on the idea of seeking extreme values. For discrete variables, marginal analysis is generally used to find extreme values; if it is a continuous variable, differentiation is required to find the extreme value; for complex problems, you can Use nonlinear programming or dynamic programming.

\subsubsection{Optimized configuration of charging station}

The charging station service system is the standard $M / M / c$ model. With the minimum total cost of the charging service system per unit time as the goal, the optimal cost model of the charging service system is established as follows:

$$
\min S=s_{1} c+s_{2} L_{s}
$$

Where:

$S_{1}$ — The total service cost corresponding to a single charging pile per unit time;

$s_{2} \_$Expenses incurred by a single electric vehicle per unit time due to staying;

$L_{s} \_$Average captain of charging service system;

$s_{1} c$ - The total cost of the charging station per unit time;

$s_{2} L_{s}$ Expenses incurred by users due to staying and waiting per unit of time.

The specific formula of the total service cost $s_{1}$ corresponding to a single charging pile per unit time is:

ISSN: 0010-8189

(C) CONVERTER 2020

www.converter-magazine.info 


$$
S_{1}=S_{11}+S_{12}
$$

Where:

$S_{11}$ - Indicates the fixed construction cost corresponding to a single charging pile converted to a unit time;

$S_{12}$ Indicates the operating cost of a single charging pile per unit time, including workers' wages, equipment maintenance fees, etc;

$S_{11} 、 S_{12}$ The specific formula is as follows:

$$
\left\{\begin{array}{l}
s_{11}=\frac{1}{c} \frac{1}{8760} \frac{1}{m} F_{1} \\
s_{12}=\frac{1}{c} \frac{1}{8760} F_{2}
\end{array}\right.
$$

Where:

$F_{1}$ _- Indicates the total cost including the cost of purchasing distribution transformers, charging piles, and construction costs;

$m-$ Indicates the expected operating life;

$F_{2}$ _-Indicates the total annual cost of workers' wages, equipment maintenance, etc;

If $\mathrm{c}$ is the optimal value of the number of charging piles in the charging station, the total cost of the charging service system is the smallest at this time, and the marginal analysis method is used to obtain:

$$
\left\{\begin{array}{l}
S(c) \leq S(c-1) \\
S(c) \leq S(c+1)
\end{array}\right.
$$

The formula (7) is substituted into the following formula (10), and both sides of the two formulas are divided by $s_{2}$, which can be sorted out:

$$
L_{s}(c)-L_{s}(c+1) \leq \frac{s_{1}}{s_{2}} \leq L_{s}(c-1)-L_{s}(c)
$$

The actual meaning of $\mathrm{c}$ is an integer, so as long as we substitute different values of $\mathrm{c}$, we can get the value of $\mathrm{c}$ that satisfies the formula (11), which is the optimal solution of the objective function.

2.4.2. Scale adjustment of charging stations based on the bearing capacity of the distribution network After obtaining the optimal number of charging piles, we need to compare the total power of the distribution network in the area with the total power of the charging station for the sake of regional power safety considerations, so as to avoid the excessive power consumption that causes the regional grid to be unbearable. malfunction. Assuming that the regional distribution network can accept the maximum power as $P_{\max }$; the charging power of the charging pile is $\mathrm{P}$, then the maximum power of the charging station is $P \cdot c$.

If $P \cdot c \leq P_{\max }$, the service radius of the charging station and the configuration of the charging piles in the station are optimal; if $P_{\max } \leq P \cdot c$, it means that the regional distribution network cannot bear it, and the service radius and scale of the charging station need to be adjusted. 
When $P_{\max } \leq P \cdot c$, the maximum number of charging piles that the charging station can set is:

$$
c^{\prime}=\left[\frac{P_{\max }}{P}\right]
$$

where the symbol [] means rounding down.

According to the actual situation of electric vehicles going to the charging station for charging, it can be considered that the number of electric vehicles served by the charging pile is the same at the same time. Then the adjusted number of electric vehicles is:

$$
N^{\prime}=\frac{c^{\prime}}{c} N
$$

According to formula (5), the adjusted service radius of the charging station is obtained.

\section{The example analysis}

In this paper, we select Haishu District of Ningbo City as the research object of charging stations.

3.1. Area selection and data preparation of charging stations

We take the second ring road in the main city of Haishu District, Ningbo City as the research object. We first get the topology of the study area, and obtain some points A to K, which are both candidate charging stations and demand points . Record the distance between each point as shown in Table 1:

Table 1 Distance between candidate points

\begin{tabular}{c|c|c|c|c|c|c|c|c|c|c|c}
\hline $\mathrm{km}$ & $\mathrm{A}$ & $\mathrm{B}$ & $\mathrm{C}$ & $\mathrm{D}$ & $\mathrm{E}$ & $\mathrm{F}$ & $\mathrm{G}$ & $\mathrm{H}$ & $\mathrm{I}$ & $\mathrm{J}$ & $\mathrm{K}$ \\
\hline $\mathrm{A}$ & 0 & 4.7 & 6.9 & 6.9 & 11.6 & 8.9 & 7 & 6.2 & 4.5 & 6.4 & 2.3 \\
\hline $\mathrm{B}$ & 4.7 & 0 & 2.6 & 3 & 8.4 & 6.7 & 8.9 & 4.8 & 3.6 & 6.3 & 3 \\
\hline $\mathrm{C}$ & 6.9 & 2.6 & 0 & 4.1 & 8 & 8.1 & 10.5 & 6.3 & 5.2 & 7.7 & 4.9 \\
\hline $\mathrm{D}$ & 6.9 & 3 & 4.1 & 0 & 5.4 & 5 & 8 & 4 & 3.3 & 5.7 & 4.6 \\
\hline $\mathrm{E}$ & 11.6 & 8.4 & 8 & 5.4 & 0 & 2.7 & 4.6 & 5.4 & 7.1 & 6.5 & 9 \\
\hline $\mathrm{F}$ & 8.9 & 6.7 & 8.1 & 5 & 2.7 & 0 & 3.5 & 2.8 & 4.5 & 4 & 6.4 \\
\hline $\mathrm{G}$ & 7 & 8.9 & 10.5 & 8 & 4.6 & 3.5 & 0 & 4.2 & 5.2 & 3 & 6.3 \\
\hline $\mathrm{H}$ & 6.2 & 4.8 & 6.3 & 4 & 5.4 & 2.8 & 4.2 & 0 & 2.2 & 1.8 & 3.7 \\
\hline $\mathrm{I}$ & 4.5 & 3.6 & 5.2 & 3.3 & 7.1 & 4.5 & 5.2 & 2.2 & 0 & 2.6 & 2 \\
\hline $\mathrm{J}$ & 6.4 & 6.3 & 7.7 & 5.7 & 6.5 & 4 & 3 & 1.8 & 2.6 & 0 & 3.7 \\
\hline $\mathrm{K}$ & 2.3 & 3 & 4.9 & 4.6 & 9 & 6.4 & 6.3 & 3.7 & 2 & 3.7 & 0 \\
\hline
\end{tabular}

Assuming that the maximum time $T$ for a user in Ningbo to reach an electric vehicle charging station is 10 minutes, the average vehicle speed $V$ is $30 \mathrm{~km} / \mathrm{h}$, and the traffic impedance coefficient $\alpha$ is 1.3 , then the service radius $R$ of the charging station can be calculated as $3.83 \mathrm{~km}$ according to formula (1).

ISSN: 0010-8189 
Then using the 0-1 planning method in integer planning, we require at least one charging station for each demand point within the range of $R$ to ensure that the user can charge and power, which can be expressed by the following formula

$$
\begin{aligned}
& \text { Min } z=A+B+C+D+E+F+G+H+I+J+K \\
& \left(\begin{array}{l}
(1) A+K>=1 \\
(2) B+C+D+I+K>=1 \\
(3) B+C>=1 \\
(4) B+D+I>=1 \\
(5) E+F>=1 \\
(6) E+F+G+H>=1 \\
(7) F+G+J>=1 \\
(8) F+H+I+J+K>=1 \\
(9) B+D+H+I+J+K>=1 \\
(10) G+H+I+J+K>=1 \\
(11) A+B+H+I+J+K>=1 \\
A, B \ldots \ldots . K=0 \text { or } 1
\end{array}\right.
\end{aligned}
$$

Put the formula into MATLAB and get the result. It can be obtained that the charging station will be built at points $\mathrm{B}, \mathrm{F}$, and $\mathrm{K}$.

\subsection{Constant capacity optimization of charging station}

Set the parameters according to the actual situation of electric vehicles on the Chinese market in recent years:

The average annual mileage of the vehicle is $40000 \mathrm{~km}$;

The energy consumption of a vehicle per 100 kilometers is $15 \mathrm{kWh}$;

The vehicle battery capacity is $20 \mathrm{kWh}$;

The average annual driving days of the vehicle is $240 \mathrm{~d}$.

The study area is within the second ring road, which is basically the urban area. For the convenience of research, the area car density $\mathrm{f}$ is set to 100 , and the number of cars $N$ in the service area of a charging station is 4608 , and then the number of cars $N$ is substituted into the formula (14), the number of electric vehicles arriving at the charging station per unit time $\lambda=240$. Suppose the average service rate of each charging pile $\mu=50$, the charging station has c charging piles, and the average utilization rate of the system service organization $\rho=4.8 / \mathrm{c}$ According to the above, the time interval for electric vehicles to reach the charging station obeys the parameters Is the Poisson distribution of $\lambda$, and the time of receiving the service of the charging pile obeys the negative exponential distribution of parameter $\mu$. In this era, the number $c$ of different service stations is entered into formula (3) and formula (4), and the results of the system indicators are shown in Table 2:

Table 2 Results of different c system indicators

\begin{tabular}{c|c|c}
\hline c value & $P_{0}(c)$ & $L_{s}(c)$ \\
\hline 1 & -3.799392 & -1.2622 \\
\hline 2 & -0.411760 & -1.0083 \\
\hline 3 & -0.074626 & -1.3134 \\
\hline
\end{tabular}

ISSN: 0010-8189 
CONVERTER MAGAZINE

Volume 2021, No. 4

\begin{tabular}{c|c|c}
\hline 4 & -0.133618 & -83.8627 \\
\hline 5 & 0.001699 & 26.4408 \\
\hline 6 & 0.006096 & 6.8711 \\
\hline 7 & 0.007510 & 5.4073 \\
\hline 8 & 0.007985 & 5.0093 \\
\hline 9 & 0.008149 & 4.8744 \\
\hline 10 & 0.008204 & 4.8216 \\
\hline
\end{tabular}

It can be seen from Table 2 that when c is less than $5, L_{s}$ is negative. In other words, when $\rho \geq 1$ is in an infinite queue, only $\rho<1$ will not form an infinite queue, so we take an integer greater than 4 , Use the data in Table 2 to get Table 3:

Table 3 Results of poor system indicators

\begin{tabular}{c|c|c}
\hline c value & $L_{s}(c)-L_{s}(c+1)$ & $L_{s}(c-1)-L_{s}(c)$ \\
\hline 5 & 19.5697 & -110.3035 \\
\hline 6 & 1.4638 & 19.5697 \\
\hline 7 & 0.398 & 1.4638 \\
\hline 8 & 0.1349 & 0.398 \\
\hline 9 & 0.0483 & 0.1349 \\
\hline
\end{tabular}

According to the construction cost of the charging station published in the online article, the approximate cost data can be obtained: assuming that the cost of a charging pile is 10,000, the cost including power distribution facilities such as transformers and power distribution cabinets is 10,000, and the rest are construction costs, monitoring and other costs Ten thousand, workers' wages and maintenance equipment are ten thousand, the expected year of operation. Substituting these data into equations (8) and (9), set the cost of the user's stay as 4 , and the value of $s_{1} / s_{2}$ is shown in Table 4

Table $4 s_{1} / s_{2}$ results

\begin{tabular}{c|c|c}
\hline c value & $S_{1}$ & $S_{1} / S_{2}$ \\
\hline 5 & 13.70 & 3.43 \\
\hline 6 & 11.80 & 2.95 \\
\hline 7 & 10.44 & 2.61 \\
\hline 8 & 9.42 & 2.36 \\
\hline 9 & 8.63 & 2.16 \\
\hline
\end{tabular}

Then according to Table 3 and Table 3.4, it can be seen that when $c=6$, the charging pile of the optimal charging station is preliminarily confirmed as a station. Its system indicators are shown in Table:

Table 5 System index results at $c=6$

\begin{tabular}{c|c|c|c}
\hline System indicators & Value & System indicators & Value \\
\hline$L_{q}$ & 2.0711 & $T_{q}$ & 0.0863 \\
\hline$L_{s}$ & 6.8711 & $T_{s}$ & 0.2863 \\
\hline
\end{tabular}

3.3. Adjust the charging station based on the maximum withstand power of the distribution network

ISSN: 0010-8189 
Assuming that the maximum power $P_{\max }$ of the distribution network in a single charging station area is $520 \mathrm{~kW}$, and the rated power of each charging pile is $100 \mathrm{~kW}$, then the power of the charging station is $600 \mathrm{~kW}$, $P \cdot c>P_{\max }$, so it needs to be re-adjusted. According to formula (12), we can see that The adjusted number of charging piles is 5 .

According to formula (21), the number of cars that can be serviced after adjustment is 3840 . At this time, we substitute $N^{\prime}$ into formula (5) and formula (6) to obtain a new service radius $R^{\prime}$ of $3.497 \mathrm{~km}$ and $\lambda$ of 200 . At this time, we once again use the queuing model, substituting the data into equations (3) and (4), and get the indicators as shown in Table 6:

Table 6 System index results

\begin{tabular}{c|c|c|c}
\hline System indicators & Value & System indicators & Value \\
\hline$L_{q}$ & 2.21645 & $T_{q}$ & 0.11082 \\
\hline$L_{s}$ & 6.21645 & $T_{s}$ & 0.31082 \\
\hline
\end{tabular}

Then need to verify whether the formula (11) is satisfied, we get the calculation as shown in Table 7:

Table $7 \mathrm{c}$ is the system index results at 4,5 , and 6 o'clock

\begin{tabular}{c|c|c}
\hline c value & $P_{0}(c)$ & $L_{s}(c)$ \\
\hline 4 & 0.01298 & $\infty$ \\
\hline 5 & 0.01298 & 6.2165 \\
\hline 6 & 0.01668 & 4.5695 \\
\hline
\end{tabular}

According to the table, when c $=5, L_{s}(5)-L_{s}(6)=1.647, L_{s}(4)-L_{s}(5)=\infty$, so the formula (11) is still satisfied when $\mathrm{c}=5$.

The service radius of the adjusted charging station is $3.497 \mathrm{~km}$, and the scale is 5 charging piles. Now that the service radius has changed, it is necessary to plan the site selection 0-1 again, and the other conditions except the service radius remain unchanged. At each demand point, there is at least one charging station within the range of $R$ to ensure that users can charge. It can be expressed by the following formula: 


$$
\left\{\begin{array}{l}
(1) \mathrm{A}+\mathrm{K}>=1 \\
(2) \mathrm{B}+\mathrm{C}+\mathrm{D}+\mathrm{K}>=1 \\
(3) \mathrm{B}+\mathrm{C}>=1 \\
(4) \mathrm{B}+\mathrm{D}+\mathrm{I}>=1 \\
(5) \mathrm{E}+\mathrm{F}>=1 \\
(6) \mathrm{E}+\mathrm{F}+\mathrm{H}>=1 \\
(7) \mathrm{G}+\mathrm{J}>=1 \\
(8) \mathrm{F}+\mathrm{H}+\mathrm{I}+\mathrm{J}>=1 \\
(9) \mathrm{D}+\mathrm{H}+\mathrm{I}+\mathrm{J}+\mathrm{K}>=1 \\
(10) \mathrm{G}+\mathrm{H}+\mathrm{I}+\mathrm{J}>=1 \\
(11) \mathrm{A}+\mathrm{B}+\mathrm{I}+\mathrm{K}>=1 \\
\mathrm{~A}, \mathrm{~B}, \ldots \ldots . . \mathrm{K}=0 \text { or } 1
\end{array}\right.
$$

Put Equation 14 into MATLAB for calculation, and get the result. The result is that the charging station construction sites are points A, B, E, and J.

\section{Conclusion}

In the 21 st century with serious environmental pollution, which technology can save energy and protect the environment, then it will receive widespread attention, will be vigorously promoted, and will be able to develop rapidly. Therefore, as a new energy vehicle, electric vehicles have very good development prospects. In recent years, my country has issued many support policies for new energy vehicles, which has allowed the rapid development of electric vehicles in my country. In this way, the construction of charging is particularly important, but we cannot blindly construct it. The construction of charging stations needs to consider many factors, such as where the charging station is built, how many electric vehicle customers can be served, and how many charging piles are equipped in the charging station. The problem is of practical significance, so it is urgent to study the location and capacity of charging stations.

This paper studies the simulation problem of charging stations in Haishu District of Ningbo City. First, the central place theory is used to determine the service radius based on the convenience of users. The 0-1 planning is used to initially select the simulation point, and then the queuing theory is adopted as Based on the basic theory, establish the charging station queuing model, and then use the minimum service cost in the unit as the objective function to solve the optimal number of charging piles. On this basis, compare the bearing capacity of the distribution network to adjust the number of charging piles at the charging station. We can determine the service radius of the charging station and the optimal number of charging piles, once again use the 0-1 plan, and finally determine the site to be built.

\section{References}

[1] Tang M. Gong D. Liu S. Finding Key Factors Affecting the Locations of Electric Vehicle Charging Stations: a Simulation and ANOVA Approach. 2017, 16(3): 541-554.

[2] Narges Shahraki, Hua Cai, Metin Turkay, Ming Xu. Optimal locations of electric public charging stations using real world vehicle travel patterns. Transportation Research Part D, 2015, 41(5): 165176. 
[3] Wang Meng, Liu Kai. Location of Electric Vehicle Charging Station Based on Spatial Clustering and Multi-hierarchical Fuzzy Evaluation. Transactions of Nanjing University of Aeronautics and Astronautics, 2017, 34(01): 89-96.

[4] Chengzhang Wang, Fang He, Xi Lin, Zuo-Jun Max Shen, Meng Li. Designing locations and capacities for charging stations to support intercity travel of electric vehicles: An expanded network approach. Transportation Research Part C, 2019, 102(8): 210-232.

[5] Xianqiang Ren, Huiming Zhang, Ruohan Hu, Yueming Qiu. Location of electric vehicle charging stations: A perspective using the grey decision-making model. Energy, 2019, 173(7): 548-553.

[6] Jia Xu, Jing Li, Xin Liao. A Prediction Method of Charging Station Planning Based on BP Neural Network. 2019, 7(7): 219-230.

[7] Jing-min Wang, Yan Liu, Yi-fei Yang, The Location of Electric Vehicle Charging Stations based on FRLM with Robust Optimization. 2019, 33(8): 19-19.

[8] Rui Chen, Xinwu Qian, Lixin Miao, Satish V, Ukkusuri. Optimal charging facility location and capacity for electric vehicles considering route choice and charging time equilibrium. Computers and Operations Research, 2020, 113(9): 112-136.

[9] Xue Bai, Kwai-Sang Chin, Zhili Zhou. A bi-objective model for location planning of electric vehicle charging stations with GPS trajectory data. Computers \& Industrial Engineering, 2019, 128(4): 591604.

[10] Bing Han. Research on location selection of washing and dyeing industry facilities. Beijing, China Agricultural University, 2007.

[11] Yahui Song. Research on the layout planning of urban electric vehicle charging facilities. Beijing Jiaotong University, 2011. 